\title{
GCU
}

Glasgow Caledonian

University

University for the Common Good

\section{Estimating the population size of people who inject drugs in Malaysia for 2014 and 2017 using the benchmark-multiplier method}

Hiebert, Lindsey; Azzeri, Amirah; Dahlui, Maznah; Hecht, Robert; Mohamed, Rosmawati; Shabaruddin, Fatiha Hana; McDonald, Scott A.

Published in:

Substance Use and Misuse

DOI:

10.1080/10826084.2019.1708943

Publication date:

2020

Document Version

Author accepted manuscript

Link to publication in ResearchOnline

Citation for published version (Harvard):

Hiebert, L, Azzeri, A, Dahlui, M, Hecht, R, Mohamed, R, Shabaruddin, FH \& McDonald, SA 2020, 'Estimating the population size of people who inject drugs in Malaysia for 2014 and 2017 using the benchmark-multiplier method', Substance Use and Misuse, vol. 55, no. 6, pp. 871-877.

https://doi.org/10.1080/10826084.2019.1708943

\section{General rights}

Copyright and moral rights for the publications made accessible in the public portal are retained by the authors and/or other copyright owners and it is a condition of accessing publications that users recognise and abide by the legal requirements associated with these rights.

Take down policy

If you believe that this document breaches copyright please view our takedown policy at https://edshare.gcu.ac.uk/id/eprint/5179 for details

of how to contact us. 
Estimation of the population size of people who inject drugs in Malaysia for 2014 and 2017 using the benchmark-multiplier method

\begin{abstract}
Background. As hepatitis C elimination efforts are launched, national strategies for screening and treatment scale-up in countries, such as Malaysia, must be designed and implemented. Strategic information, including estimates of the total number of patients chronically-infected with hepatitis $\mathrm{C}$ virus (HCV) and the size of key populations, such as people who inject drugs (PWID), is critical to informing these efforts. For Malaysia, the estimate of the PWID population size most frequently reported in global systematic reviews is for the year 2009.

Objectives. To support ongoing national HCV planning efforts, we aimed to estimate the national population size of active PWID in Malaysia, for the years 2014 and 2017.

Methods. To estimate the PWID population size, we applied standard benchmark-multiplier methodology, frequently used for PWID population size estimation, and extended it by adjusting for cessation of injecting drug use within the benchmark and calculating statistical uncertainty intervals.
\end{abstract}

$\underline{\text { Results. }}$ The estimated active PWID population size was 153,000 (95\% uncertainty interval (UI): 136,000-172,000) for 2014 and 156,000 (95\% UI: 137,000-188,000) for 2017.

Conclusions/importance. This updated estimate of the active PWID population size in Malaysia will help inform effective planning for the scale-up of HCV screening and treatment services. The proposed methodology is applicable to other countries that maintain national HIV registries and have conducted Integrated Biological and Behavioral Surveys among active PWID.

\title{
KEYWORDS
}

Injecting drug use; Malaysia; HIV; population estimation 


\section{Introduction}

The WHO Global Health Sector Strategy (GHSS) for hepatitis has set diagnosis and treatment targets for elimination of chronic hepatitis C virus (HCV) infection (by 2030)(WHO, 2016). As Malaysia seeks to scale-up HCV awareness, screening, and treatment programs, up-to-date estimates of the sizes of the populations to be supported and targeted are vital for purposes of planning and resource allocation. In the Malaysian setting, one of these key populations is people who inject drugs (PWID). National-level chronic HCV prevalence among persons aged 15-64 years in 2009 was estimated at $1.8 \%$ (95\% CI: 1.6-2.2\%), with an estimated 59\% of cases having a history of injection drug use (McDonald et al., 2014). It is also estimated that about $63-67 \%$ of active PWID in Malaysia have been exposed to HCV and thus test HCV antibody-positive (Ali et al., 2018; Vicknasingham et al., 2009).

The denominator of this prevalence value - the size of the active PWID population (we treat the PWID population as a simple division into PWID who either have, or have not, permanently ceased injecting; the latter group we term 'active' PWID) - has been estimated on a number of occasions since the mid-1990s (Reid et al., 2004), with the most commonly cited value estimated for the year 2009. This estimate of the active PWID population size $(170,000)$ was derived using expert consensus based on various published estimates (Ministry of Health Malaysia, 2014), and uncertainty around this figure had not been quantified.

Given the importance of this risk group to the overall burden of HCV (and HIV), a recent, valid estimate of the size of the active PWID population is a crucial input for understanding the full epidemiological landscape and for strategic planning to expand testing and antiviral treatment services to achieve the WHO targets. There is also some indication of a decreasing trend in this 
population size; for instance new client registrations at NGO-run needle/syringe exchange programme (NSEP) service points declined from 2013 to 2015 (Chandrasekaran et al., 2017).

To obtain this population size estimate, household surveys are considered unreliable because injection drug use is a covert and stigmatized behaviour. PWID are less likely to have stable living arrangements, which means they have a lower chance of being captured by routine sampling methods, they are less likely to respond to these surveys if sampled, and if they respond they are more likely to underreport such behaviours (UN, 2013; Hickman \& Taylor, 2005). These trends (unreliability of survey methods) are exacerbated in societies where injection drug use is criminalized. Historically, Malaysia has some of the most punitive drug laws in the world, and thus survey methods would not be effective in this context.

Therefore, to estimate the population size of 'hidden' populations, indirect methodologies are often used. Indirect estimation involves using one data source as a sample of the population of interest and then another data source to estimate the sampling intensity of this sample: the proportion of the population represented in the sample. Important assumptions for indirect methods include a relatively stable population and data sources that are sufficiently representative of the population for which size is being estimated (Hickman \& Taylor, 2005). One of the most convenient indirect approaches is the benchmark-multiplier method; this methodology relies on existing data sources, and has been used for PWID population size estimation in Australia, Belgium and India, among others (Hickman et al., 2002; Bollaerts et al., 2013; Larney et al., 2017; Medhi et al, 2012).

Our main objective was to estimate the current national population size of active PWID in Malaysia for the year 2017. Using the same approach and data sources, we also estimated the population size for 2014 to determine if there was any evidence for a change over this period. 
Building on the standard benchmark-multiplier methodology, we additionally adjust for cessation of injecting drug use amongst the benchmark using modelling methods, and estimate statistical uncertainty intervals.

\section{Methods}

\section{Benchmark-multiplier method}

The benchmark-multiplier method (hereafter, multiplier method) is an established, indirect method for population size estimation that is useful when the population of interest is difficult to reach (Hickman et al., 2002; Bollaerts et al., 2013; Larney et al., 2017). Estimation of the size of PWID populations is inherently challenging, as definitions must be formalised (such as current/active or recent injector, vs. ex-injector) and treatment of potential biases in the data needs to be considered (Sweeting et al., 2009; Larney et al., 2015). In this approach, only two parameters are required: the benchmark and the multiplier. The size of the population of interest can be calculated by Population size $=$ benchmark * (1/multiplier $).$ The benchmark enumerates, at the population level, all persons within a particular (vulnerable) population who engage with a specific institution or service, or have a certain attribute (such as seropositive status). The multiplier is a factor derived from the prevalence of attending that service/institution or possessing the same attribute among a random sample of the population of interest.

Advantages of this method are that it can be more reliable than a census or enumeration approach, and that the types of data drawn upon are flexible. The principal limitation of the multiplier method is that accuracy heavily depends on the quality of the data, namely completeness of the benchmark and representativeness of the multiplier. To ensure accurate estimation, the data sources should be obtained independently, have aligned age ranges, aligned time periods, and be comparable in geographic scope. 


\section{Defining the parameters}

The benchmark parameter was defined as the number of people living with HIV (PLHIV) who are active PWID. The multiplier was defined as the prevalence of HIV infection among a sample of active PWID. To explore changes in population size over time, we estimated the active PWID population size for 2014 and 2017; therefore two year-specific values for the benchmark and two for the multiplier parameter were required.

\section{Data sources}

To estimate the benchmark we relied on national HIV surveillance data. The number of PLHIV in Malaysia, aggregated over all infection acquisition routes, is routinely published by the Ministry of Health (MOH) Malaysia within Global AIDS Response Progress Reports (e.g., Ministry of Health Malaysia, 2012; 2015a; 2016). These reports present standard indicators derived from the national HIV/AIDS surveillance system, which evolved from a manual-based notification to electronic notification to a web-based National AIDS registry in 2009. Although the HIV surveillance system contains annual notifications stratified by risk group, in only one of the recent Progress Reports was the PLHIV figure separately reported for injecting drug use (IDU) risk - for the year 2011 (Ministry of Health Malaysia, 2012). The PLHIV figures for subsequent years were therefore estimated through statistical modelling based on HIV surveillance data on the annual number of new HIV cases with IDU risk. This involved considering death as a binomial process. The timeindependent annual probability of death $(\mu)$ was first fit using the data from 1986 through 2011 (Bollaerts et al., 2013); we then forward-projected the annual numbers of PLHIV with IDU risk for 2012 to 2017 using this fitted mortality probability. The number surviving in year $j$ from each 'cohort' of new HIV cases notified in year $i\left(N_{i}\right)$ is a function of the mortality probability $\mu$ and the number of years elapsed since notification: 


$$
\text { Survived }_{i, j}=N_{i}(1-\mu)^{j-i+1}
$$

Annual numbers of new HIV cases with IDU risk were sourced from Global AIDS Response Progress Reports and MOH web publications (Ministry of Health Malaysia, 2012; 2015a; 2016; 2018a). The annual mortality probability was estimated at $1.37 \%$. The projected number of people living with HIV who are active PWID in the period 2012-2017 are shown in Table 1.

The multiplier, the prevalence of HIV infection among a sample of active PWID, was obtained from the prevalence of HIV infection among Integrated Biological and Behavioral Survey (IBBS) respondents (Ministry of Health Malaysia, 2015b; Ramly, 2018). The IBBS is a survey carried out every two to three years among active PWID and other vulnerable populations using respondentdriven sampling scheme (Gile \& Handcock, 2010). We selected multipliers using the two most recent IBBS years, 2014 and 2017, and defined benchmarks for these two years (see below). The IBBS study populations are considered to be samples of active PWID; the criteria for inclusion was 18 years or over and a minimum injecting duration of 6 months, with no constraint on frequency of injecting. In the 2014 survey, $71 \%$ reported injecting at least once per day.

\section{Estimation of the cessation of injecting}

The benchmark can be considered to enumerate the population of HIV-positive ever-PWID; however, many of those with reported IDU risk may have permanently ceased injecting before or after testing/notification. As the multiplier is obtained from a survey among active injectors, and our research objective was to estimate the active PWID population size, it was essential to adjust 
the benchmark for cessation. Therefore, we required an estimate of the proportion of ever-PWID who had permanently ceased injecting, taking into account the competing risk of mortality.

To estimate the proportion of all living PWID who had permanently ceased injecting as of 2014 and 2017, we employed a simple modelling approach. We adapted a multi-state Markov model of the Malaysian PWID population dynamics from previous work (McDonald et al., 2014), but restricted the modelled population to HIV-positive PWID who can be seen as occupying one of three states: Active PWID, Ex-PWID, and Dead (see Supplementary Materials Fig. S1). In this model, the parameters describing the rates, or transition probabilities, by which persons move between the various states are: (permanently) ceasing to inject, and mortality. The inflow is the annual number of HIV seroconversions among active PWID (see Supplementary Materials for further details).

The number of PLHIV in a particular year who are still active injectors could then be calculated as the estimated total number of people living with HIV who have a history of injection drug use (as of that year; see benchmark description above), reduced by the proportion estimated to have permanently ceased injecting as of that year.

\section{Computation of the active PWID population size}

Computation was done using Monte-Carlo simulation, which allowed statistical uncertainty in the population size estimates to be expressed. The following fully describes the statistical model:

$$
\begin{gathered}
m_{i} \sim \operatorname{Binomial}\left(M_{i}, \pi_{i}\right) \\
N_{i}=\frac{B_{i}}{\pi_{i}}
\end{gathered}
$$


In the above, $N$ denotes the population size to be estimated, $M$ and $m$ refer to the denominator (active PWID survey respondents) and the numerator (HIV seropositives) of the multiplier data, respectively; $B$ denotes the benchmark parameter (PLHIV with IDU risk, adjusted for cessation of injection), and index $i$ refers to the year of estimation. We could only produce estimates for 2014 and 2017 as survey data for the multiplier parameter were only available for these years. The multiplier was defined using 'crude' prevalence (i.e., total positive HIV tests divided by total respondents), but we note that determining prevalence from respondent-driven sampling (RDS) data requires estimators that take the nature of RDS sampling into account (Bazazi et al., 2015).

Prevalence of active PWID per 1,000 population aged 15-64 years was calculated using 2017 national population estimates (IndexMundi, 2018), which was 20,736,942 for both sexes and 10,501,664 for males only. The code to produce the estimates was implemented in R version 3.5.1 (R Core Team, 2018).

\section{Sensitivity analysis}

Because the estimated active PWID population size depends on the cessation rate/injecting duration parameter (Sweeting et al., 2009) and we lacked satisfactory data for estimating this, we

also conducted sensitivity analyses using (i) a shorter duration of injecting (based on studies from the Asian region within a recent systematic review (Fazito et al., 2012), which employed a heuristic method of doubling the median reported duration, yielding 8.8 years); and (ii) a longer injecting duration (the IBBS-2012 value of 11.7 years (Ministry of Health Malaysia, 2012), derived using the same 'doubling' heuristic, yielding an average duration of 23.5 years).

\section{Validation exercise}


Ideally, we would prefer to synthesize evidence from more than one data source informing the active PWID population size. A logical second benchmark-multiplier set would be methadone maintenance therapy (MMT) service data (Larney et al., 2017). Methadone was piloted as a primary treatment of opioid dependence in 2005 and buprenorphrine in 2006 . The government committed to scaling up MMT in 2006 as a response to the HIV epidemic and the large PWID population (Ali et al, 2008). Over time, MMT was expanded to government-accredited health facilities (hospitals, health clinics, drug treatment centres), private GPs, civil society-operated projects, and prisons (Vijay et al., 2015).

Although data to inform the multiplier - the proportion of (ever) MMT contact among active PWID - could be located (Ministry of Health Malaysia, 2015b), the appropriate data to derive the prevalent number of active MMT clients - the snapshot number of clients on a given day (Larney et al., 2017) - is not available. However, as the cumulative number of registered MMT clients has been reported (Ministry of Health Malaysia, 2016), we could apply the multiplier method to estimate a lower bound on the population size of living ever-PWID, and thus use MMT data as an approximate validation.

Here, the multiplier is the proportion of IBBS-2014 respondents who ever enrolled in MMT (34.5\%)(Ministry of Health Malaysia, 2015b). We estimated the benchmark - the cumulative number of living MMT clients - by adjusting the cumulative number of registered clients as of end 2015 (approximately 88,500; Ministry of Health Malaysia, 2016; we could not locate a comparable figure for a more recent year) for mortality by applying a $8.6 \%$ mortality proportion (Ali et al., 2018). The cumulative number of living MMT clients (the benchmark) was estimated at $78,100$. 
Because only the cumulative (as opposed to current) number of living MMT clients was available, we can only validate the estimate derived using the MMT client benchmark against the cumulative number of PLHIV who ever injected drugs. We estimated the latter figure (as of end 2015) using benchmark-multiplier methods as described above to adjust for mortality, but not correcting for cessation of injecting. For the ever-PWID population size estimate, the benchmark was 55,778 (see Table 1). For the multiplier, we defined lower and upper bounds by imposing a range, corresponding to the highest and lowest observed HIV seroprevalence among active PWID (22.1\% and $13.5 \%$, from IBBS-2009 and IBBS-2017, respectively).

\section{Results}

Mean active PWID population size estimates for 2014 and 2017, with 95 percent uncertainty intervals, were 153,000 (95\% UI: 136,000-172,000) and 156,000 (95\% UI: 137,000-178,000), respectively (Table 1). For 2017, this corresponds to an active PWID prevalence of 7.5 per 1,000 population aged 15-64 years. Under the assumption that 3.7\% of active PWID are female (Bazazi et al., 2015), then the male-only active PWID prevalence is estimated at 14.3 per 1,000 among persons aged 15-64 years. Given that the 95\% intervals around the estimated population size for the two estimation years largely overlapped, there was no indication of a change over this threeyear period.

[Table 1 about here]

Exploration of the sensitivity of active PWID population size to average injecting duration indicated that our estimates were highly sensitive to this parameter. The shorter average duration (8.8 years) yielded median population size estimates of 99,000 and 94,000 for 2014 and 2017, 
respectively. Assuming a longer injecting duration (23.5 years) resulted in much higher estimated active PWID population sizes of 198,000 and 212,000 for 2014 and 2017, respectively.

\section{Validation}

Combining the cumulative number of living MMT clients (the benchmark) with the proportion of active PWID in 2014 who reported being on MMT (multiplier) gave an approximate lower bound on living ever-PWID as of end 2015 of $226,000(78100 * 1 / 0.345)$. This figure we deemed a lower bound because the MMT enrolment proportion among the entire population of PWID (active and ex-PWID) is almost certainly smaller than $34.5 \%$, due to the limited number of MMT facilities at the time of introduction of harm reduction services.

This lower bound $(226,000)$ is roughly consistent with the ever-PWID population size estimate as of end 2015; this estimate ranged from 252,000 to 413,000 (range calculated as [55778 * 1/0.221] and $[55778 * 1 / 0.135]$, respectively). Note that this estimate depends on the very strong assumption that HIV prevalence obtained from these relatively recent IBBS surveys is representative of overall prevalence since the beginning of HIV epidemic; i.e., the prevalence among both active PWID and ex-PWID who are alive at the end of 2015.

\section{Discussion}

This updated, recent estimate of the national population size of people who actively inject drugs in Malaysia (156,000 for 2017; 95\% UI: 137,000-178,000) will be useful in academic, governmental, civil society, and clinical settings to guide planning and resource allocation for Malaysia's hepatitis $\mathrm{C}$ elimination strategy. Our methodology offers an improvement upon approaches to determine PWID population size using the multiplier method in which the multiplier is derived from HIV seroprevalence data from active PWID and the benchmark is derived from registration 
of HIV cases with IDU risk, but without accounting for cessation of injecting. Such an approach that fails to adjust for cessation - would produce an estimate that lies somewhere between the size of the ever-PWID population and the size of the active PWID population.

Diminishing numbers of new client registrations have been observed at NGO-run needle/syringe exchange programme service points from 2013 to 2015 (Chandrasekaran et al., 2017). This does not, however, constitute indirect evidence for a declining active PWID population size, as other factors may influence trends in client registrations. Furthermore, we found no evidence for any change in the size of this population over the short, three-year period of 2014-2017, as point estimates for both 2014 and 2017 were very similar and 95\% uncertainty intervals extensively overlapped. We note that the estimated population-level prevalence of active PWID decreased from 0.6 per 100,000 in 2009 to 0.5 per 100,000 in 2017, in part because of population growth since 2009 .

Sensitivity analysis indicated, unsurprisingly, that the estimated active PWID population size is smaller if the average injecting career length is relatively short ( 8.8 years) compared with an assumption of a much longer (23.5 years) average career length. This is a vital parameter for which data urgently needs to be collected; this is a recognised limitation, common to previous efforts to estimate PWID population sizes in other countries (Larney et al., 2015). The validation exercise using data on cumulative MMT center clients, although approximate at best as it is subject to more severe data limitations than our main estimation, indicated consistency with the estimated ever-PWID population size, and so provided some support for our estimates of the active PWID population size. 
Since the current paper was submitted for publication, PWID population size estimates - also for the year 2017 - have been produced for Malaysia by the MOH. The first reported point estimate was 120,000 (Ministry of Health Malaysia, 2018a), but details on data sources underlying this estimate were not available. Separate estimates of 31,800 and 111,500 for male PWID were made in a subsequent exercise and extrapolated to females by assuming $2 \%$ of all PWID are female (Ministry of Health Malaysia, 2018b). The latter estimates were derived using benchmarkmultiplier methods based on HIV service data sources: PWID accessing HIV outreach services run by non-governmental organisations in the 12 month period May 2016 to April 2017, and unique clients attending MMT centers during May to July 2017, respectively.

\section{Importance of population size estimation}

The Malaysian Ministry of Health has committed to a five-year (2018-2022) HCV treatment programme using direct-acting antivirals. If there is commitment to further scale-up and effort leading to elimination beyond 2022, then next to treatment costs, the largest cost-driver of an $\mathrm{HCV}$ elimination program is likely to be screening (Hecht et al., 2018; Lim et al., 2018; World Bank, 2017). A national-level screening program design has been recently proposed to address the WHO elimination treatment goals in Malaysia using a targeted approach to limit costs during the initial phase of the programme (Hiebert et al., 2019). This step-wise screening programme initially sequentially targets various HCV high-risk sub-populations (including active PWID); after these groups are exhausted, screening in the general population would then be scaled up. The aim of this approach was to minimize costs by screening sub-populations that can be easily identified or have existing platforms, and for which the known prevalence of $\mathrm{HCV}$ is relatively high, as expected yield would be higher. The quality of the costing and evaluation of the effectiveness of this stepwise programme is highly dependent on active PWID population size; if the true population size is smaller than the 2009 estimate, expanded general population screening would be require 
implementation earlier on. Therefore, the current results will help to refine the planning and costing of a national-level HCV screening strategy.

This critical population size could not be determined by traditional household survey approaches. Injection drug use is highly stigmatized in Malaysia, and historically has been inhumanely criminalized. Under section 39B of the Malaysian Dangerous Drugs Act, those in possession of 15 gm or more of heroin and morphine, would receive a mandatory sentence of capital punishment, under the assumption that these individuals are drug traffickers (Girelli, 2019). Fortunately, these harsh drug laws are currently under governmental review, but monitoring injection drug use behaviors via population survey in Malaysia would likely suffer from extreme underreporting as individuals would unlikely to be forthcoming. Therefore, this indirect population size estimate could be critical for public health services planning and resource allocation purposes for HCV and other programs that seek to reach people who inject drugs (Hickman \& Taylor, 2005).

\section{Limitations}

The benchmark was derived from the PLHIV population size provided in routine surveillance reports by the $\mathrm{MOH}$, and was assumed accurate and to be unaffected by under-reporting. However, it is unclear if the occurrence of non-AIDS deaths have been linked to the registration system and therefore included. Moreover, not all HIV registrations had a known risk factor for acquisition. Our data was limited to those persons with IDU risk indicated; yet 9\% of new HIV cases 19862010 had unknown acquisition risk (PT Foundation, 2018). If some of these unknown risk cases were actually IDU risk - i.e., not identified as such due to stigma, awareness of drug use being a criminal offence, or other reasons - then HIV registrations with IDU risk will be under-reported. We assumed a constant mortality rate over time, but increasing access to ART since 2006 and 
consequent improved survival may have meant under-estimation of the number of living PWID. In addition, IDU as a risk factor.

An important assumption underlying the robustness of the multiplier method is that the multiplier - obtained from the 2014 and 2017 IBBS surveys - is unbiased and representative. Survey respondents may not be representative of the entire active PWID population, as participants by definition were those PWID who were successfully approached by other respondents within the community. Those unwilling to participate may have a lower or higher risk of being HIV positive. However, the RDS method is considered to be more effective than conventional survey approaches for hard-to-reach populations, especially for those in which criminalization and stigma are inherent. We had to derive the multiplier (and associated uncertainty) solely on the reported figures, as we did not have access to the recruitment chains that would have permitted alternative estimation methodology (Gile, 2011).

Stratification by age-group or region could not be incorporated due to lack of specific data. This would be a limitation if, for instance, the multiplier yielded data for a different age range than the age range present in the benchmark; unfortunately we could not obtain age-stratified data. HIV prevalence also varies widely by region, as demonstrated by a study of PWID using RDS in three Greater Kuala Lumpur recruitment sites in 2010 (Bazazi et al., 2015), but data stratified by region or state were also not publicly available.

Finally, our model-estimated proportion of PLHIV who had permanently ceased injecting depends on directly on cessation rate assumptions, and indirectly on the mortality rate assumed among active and ex-PWID. We could not satisfactorily estimate the average duration of injection. Surveys, such as IBBS, will under-represent recent initiates to injecting, and as the surveys were conducted among active injectors only, data on reported duration of injecting are necessarily right- 
censored. As suggested by an increase in median duration of injecting drug use between IBBS2012 and IBBS-2014 surveys (11.7 and 15.0 years, respectively), the cessation rate may have also changed over time, because of ageing of long-term injectors, and/or the RDS methodology might lead to a sample biased against recent initiates. That the IBBS tends to over-represent older injectors compared with the entire active PWID population is suggested by the higher proportion of persons aged 50 years or older (18.8\%) in IBBS-2014 respondents (Ministry of Health Malaysia, 2015b) than in NSEP clients from a survey conducted in 2013-2015 (9\%; Chandrasekaran et al., 2017). As our baseline value, we used median injecting duration from IBBS-2014, which at 15 years was similar to the duration of 13 years derived from a national IBBS conducted in Vietnam and used in a recent modelling study of HCV treatment impact in the Vietnamese setting (Birger et al., 2017). Because our selected parameter value is uncertain due to the above-mentioned limitations, we also conducted sensitivity analysis using shorter and longer durations, which substantially affected the population size estimates.

\section{Conclusions}

An updated active PWID population size estimate will help inform planning of the scale-up of both HCV screening and treatment services in Malaysia, and will be of value for evaluation of prevention initiatives such as harm reduction. In the public health response to $\mathrm{HCV}$, improved strategic information can improve efficiency of resource allocation and ensure marginalised vulnerable individuals will not be overlooked. The methodology we have proposed has relevance beyond Malaysia, as it is applicable for countries that maintain national HIV case registries containing information on route of acquisition and mortality, and that have conducted Integrated Biological and Behavioral Surveys among active PWID (with accompanying HIV testing). We expect that suitable data sources exist for a number of countries in the South-East Asia and 
Western Pacific regions, allowing the current PWID population size to be estimated for these countries.

\section{ACKNOWLEDGEMENTS}

We thank the Ministry of Health Malaysia for making available the results of their 2018 key

populations estimation and for their ongoing efforts in collecting and reporting national HIV/AIDS surveillance data. The analysis and conclusions expressed in this article are those of the authors and not necessarily of other groups or individuals. 


\section{REFERENCES}

Abdul-Quader, A. S., Baughman, A. L., Hladik, W. (2014). Estimating the size of key populations: current status and future possibilities. Current Opinion HIV/AIDS, 9(2), 107-114.

Ali, N., Aziz, S. A., Nordin, S., et al. Evaluation of methadone treatment in Malaysia: Findings from the Malaysian Methadone Treatment Outcome Study (MyTOS). (2018). Substance Use Misuse, 53(2), 239-248.

Bazazi, A.R., Crawford, F., Zelenev, A., Heimer, R., Kamarulzaman, A., \& Altice, F. L. (2015). HIV prevalence among people who inject drugs in Greater Kuala Lumpur recruited using respondent-driven sampling. AIDS and Behavior, 19(12), 2347-2357.

Birger, R. B., Le, T., Kouyos, R. D., Grenfell, B. T., \& Hallett, T. B. (2017). The impact of HCV therapy in a high HIV-HCV prevalence population: A modeling study on people who inject drugs in Ho Chi Minh City, Vietnam. PLOS One, 12(5), e0177195.

Bollaerts K, Aerts M, Sasse A. (2013). Improved benchmark-multiplier method to estimate the prevalence of ever-injecting drug use in Belgium, 2000-10. Archives of Public Health, 71(1), 10.

Chandrasekaran S, Kyaw NTT, Harries AD, et al. (2017). Enrolment and retention of people who inject drugs in the Needle \& Syringe Exchange Programme in Malaysia. Public Health Action, $7(2), 155-160$.

Fazito, E., Cuchi, P., Mahy, M., \& Brown, T. (2012). Analysis of duration of risk behaviour for key populations: a literature review. Sex Transm Infect, 88(Suppl 2), i24-i32.

Gile, K. J. (2011). Improved inference for respondent-driven sampling data with application to HIV prevalence estimation. Journal of the American Statistical Association, 106(493), 135-146.

Gile KJ, Handcock,MS. (2010). Respondent-driven sampling: An assessment of current methodology. Sociol Methodol, 40(1), 285-327. 
Girelli, G. (2019). The death penalty for drug offences: Global overview 2018. London: Harm Reduction International.

Hecht, R., Hiebert, L., Spearman, W., et al. (2018). The investment case for hepatitis B and C in South Africa: adaptation and innovation in policy analysis for disease program scale-up. Health Policy and Planning, 33(4), 528-538.

Hickman, M., Taylor, C. (2005). Indirect methods to estimate prevalence. In: Sloboda, Z. (Ed.) Epidemiology of Drug Abuse. Boston: Springer.

Hickman, M., Taylor, C., Chatterjell, A., Degenhardt, L., Frischer, M., Hay, G. et al. (2002). Estimating the prevalence of problematic drug use: a review of methods and their application. Bull Narc; LIV: 15-32.

Hiebert L., Hecht R., Soe-Lin, S., Mohamed, R., Shabaruddin, F. H., Mansor, S. M. S., et al. (2019). A step-wise approach to a national hepatitis C screening strategy in Malaysia to meet the WHO 2030 targets: Proposed strategy, coverage, and costs. Value in Health Regional. IndexMundi (2018). Malaysia Demographics Profile 2018. https://www.indexmundi.com/malaysia/demographics_profile.html. Accessed 6 November 2018. Larney, S., Grebely, J., Hickman, M., De Angelis, D., Dore, G. J., \& Degenhardt, L. (2015). Defining populations and injecting parameters among people who inject drugs: Implications for the assessment of hepatitis C treatment programs. International Journal of Drug Policy, 26(10), 950-957.

Larney S., Hickman, M., Guy, R., et al. (2017). Estimating the number of people who inject drugs in Australia. BMC Public Health, 17(1), 757.

Lim, A. G., Qureshi, H., Mahmood, H., et al. (2018). Curbing the hepatitis C virus epidemic in Pakistan: the impact of scaling up treatment and prevention for achieving elimination. International Journal of Epidemiology, 47(2), 550-560. 
McDonald, S. A., Mohamed, R., Dahlui, M., Naning, H., Kamarulzaman A,. (2014). Bridging the data gaps in the epidemiology of hepatitis $\mathrm{C}$ virus infection in Malaysia using multi-parameter evidence synthesis. BMC Infectious Diseases, 14(1), 564.

Medhi, G. K., Mahanta, J., Akoijam, B. S., Adhikary, R. (2012). Size estimation of injecting drug users (IDU) using multiplier method in five districts of India. Subst Abuse Treat Prev Policy. 2012;7:9.

Ministry of Health Malaysia (2012). Global AIDS Response Country Progress Report Malaysia 2012. Putrajaya, Malaysia: Ministry of Health.

http://www.moh.gov.my/images/gallery/Report/GLOBAL_AIDS_Endorsed_DG.pdf. Accessed 13 November 2018.

Ministry of Health Malaysia, HIV/STI Section. (2014). Malaysia 2014 Country Responses to HIV/AIDS: Reporting Period January 2013 to December 2013. Kuala Lumpur, Malaysia: Ministry of Health.

Ministry of Health Malaysia (2015a). Global AIDS Response and Progress Report, Malaysia. Putrajaya, Malaysia: Ministry of Health.

http://www.unaids.org/sites/default/files/country/documents/MYS_narrative_report_2015.pdf. Accessed 13 November 2018.

Ministry of Health Malaysia, HIV/STI/Hepatitis C Section (2015b). Integrated Biological \& Behavioral Surveillance Survey (IBBS) 2014 Report. Putrajaya, Malaysia: Ministry of Health. http://www.moh.gov.my/index.php. Accessed 13 November 2018.

Ministry of Health Malaysia. (2016). Global AIDS Response Progress Report Malaysia 2016. Putrajaya, Malaysia: Ministry of Health. http://www.moh.gov.my/images/gallery/Report/Malaysia\%20GARPR\%202016_Final.pdf. Accessed 13 November 2018. 
Ministry of Health Malaysia, HIV/STI Sector. (2012). Integrated Biological \& Behavioral Surveillance Survey (IBBS) 2012. Putrajaya, Malaysia: Ministry of Health. http://www.moh.gov.my/index.php. Accessed 13 November 2018.

Ministry of Health Malaysia, HIV/STI/Hepatitis C Sector (2018a). Country Progress Report on HIV/AIDS Malaysia 2018. Putrajaya, Malaysia: Ministry of Health.

http://www.moh.gov.my/index.php/dl\%2F554756755a584a69615852686269394d59584276636d4 6754c31567464573076556d567762334a3058306442545638794d44453458305a70626d46735879 67794b5335775a47593d. Accessed 11 April 2019.

Ministry of Health Malaysia, HIV/STI/Hepatitis C Sector. (2018b). Size of key populations in Malaysia: 2018 estimates. Putrajaya, Malaysia: Ministry of Health.

http://www.moh.gov.my/resources/index/Penerbitan/Laporan/PSE_in_Malaysia_2018.pdf. Accessed 11 March 2019.

PT Foundation. HIV/AIDS in Malaysia. http://ptfmalaysia.org/v2/hiv-sti-info/hivaids-in-malaysia/. Accessed 13 November 2018.

R Core Team. R: A language and environment for statistical computing. Vienna, Austria: R Foundation for Statistical Computing, 2018.

Ramly, M. (2018). Challenges in ending AIDS in Malaysia 2030. Unpublished presentation. Ministry of Health, Malaysia.

Reid, G., Kamarulzaman, A., \& Sran, S. K. (2004). Rapid situation assessment of Malaysia. University Malaya Department of Medicine/Infectious Disease Unit. Kuala Lumpur, Malaysia.

Sweeting, M. J., De Angelis, D., Ades, A. E., \& Hickman, M. (2009). Estimating the prevalence of ex-injecting drug use in the population. Statistical Methods in Medical Research, 18(4), 381-395. 
United Nations Office on Drugs and Crime (UN). (2003). Estimating prevalence: Indirect methods for estimating the size of the drug problem. Global Assessment Programme on Drug Abuse. Toolkit Module 2. United Nations Office on Drugs and Crime.

Vicknasingam, B., Narayanan, S., Navaratnam, V. (2009). Prevalence rates and risk factors for hepatitis C among drug users not in treatment in Malaysia. Drug Alcohol Review, 28(4), 447-454. World Bank (2017). Egypt's viral hepatitis program; burden and response: an economic analysis. Washington, DC: World Bank.

Vijay, A., Bazazi, A. R., Yee, I., Kamarulzaman, A., Altice, F. L. (2015). Treatment readiness, attitudes toward, and experiences with methadone and buprenorphine maintenance therapy among people who inject drugs in Malaysia. Journal of Substance Abuse Treatment, 54, 29-36.

World Health Organisation (WHO). Global health sector strategy on viral hepatitis 2016-2021. Geneva: WHO, 2016. 
Table 1. Data on people living with HIV (PLHIV), the estimated benchmark, the multiplier, and the mean estimated active people who inject drugs (PWID) population sizes for 2014 and 2017 (with $95 \%$ uncertainty interval (UI)).

\begin{tabular}{ccccccc} 
Year & $\begin{array}{l}\text { PLHIV } \uparrow \\
(\text { ever-PWID) }\end{array}$ & $\begin{array}{c}\text { Estimated } \\
\text { prop. ceased }\end{array}$ & $\begin{array}{c}\text { Estimated living } \\
\text { HIV+ active PWID }\end{array}$ & $\begin{array}{c}\text { Multiplier } \\
(\mathrm{n} / \mathrm{N})\end{array}$ & $\begin{array}{c}\text { Active PWID population size } \\
\text { Mean }\end{array}$ & $95 \%$ UI \\
\hline 2011 & 55891 & 0.49 & 28670 & & & \\
2012 & 56141 & 0.51 & 27510 & & & \\
2013 & 56090 & 0.53 & 26250 & & & \\
2014 & 55992 & 0.55 & 24970 & 0.163 & 152600 & $136200-172000$ \\
2015 & 55778 & 0.58 & 23650 & $(236 / 1445)$ & & \\
2016 & 55383 & 0.60 & 22320 & & & \\
2017 & 54987 & 0.62 & 21010 & $\begin{array}{l}0.134 \\
(190 / 1413)\end{array}$ & & \\
& & & & 155800 & $137100-178500$
\end{tabular}

Notes. $\uparrow 2011$ value only was available, from Ministry of Health Malaysia (2012). Values for 2012-2017 were estimated assuming a binomial process, where survival of each cohort of new HIV cases with IDU risk depends on a stable annual probability of mortality (1.37\%, determined by fitting to 1986 through 2011 data; see Methods). 